\title{
Proposal to Search for a Dark Photon in Positron on Target Collisions at DA $\Phi$ NE Linac
}

\author{
Mauro Raggi ${ }^{1}$ and Venelin Kozhuharov ${ }^{1,2}$ \\ ${ }^{1}$ Laboratori Nazionali di Frascati (INFN), Frascati, Rome, Italy \\ ${ }^{2}$ University of Sofia "St. Kl. Ohridski", Sofia, Bulgaria \\ Correspondence should be addressed to Venelin Kozhuharov; venelin.kozhuharov@cern.ch
}

Received 27 March 2014; Revised 27 June 2014; Accepted 2 July 2014; Published 22 July 2014

Academic Editor: Filipe R. Joaquim

Copyright (c) 2014 M. Raggi and V. Kozhuharov. This is an open access article distributed under the Creative Commons Attribution License, which permits unrestricted use, distribution, and reproduction in any medium, provided the original work is properly cited. The publication of this article was funded by $\mathrm{SCOAP}^{3}$.

\begin{abstract}
Photon-like particles are predicted in many extensions of the Standard Model. They have interactions similar to the photon, are vector bosons, and can be produced together with photons. The present paper proposes a search for such particles in the $e^{+} e^{-} \rightarrow$ $U \gamma$ process in a positron-on-target experiment, exploiting the positron beam of the DAФNE linac at the Laboratori Nazionali di Frascati, INFN. In one year of running a sensitivity in the relative interaction strength down to $\sim 10^{-6}$ is achievable, in the mass region from $2.5 \mathrm{MeV}<M_{U}<20 \mathrm{MeV}$. The proposed experimental setup and the analysis technique are discussed.
\end{abstract}

\section{Introduction}

The Standard Model of particle physics triumphed in 2012 with the discovery of the Higgs boson. However it is still far from consideration as the ultimate theory explaining all physical phenomena. The existence of Dark Matter is one of the examples of its failures and the search for a feasible explanation of that phenomenon is at present a major goal in particle physics.

Despite attaining the highest energy ever reached at accelerators, LHC has not been able to provide evidence for new degrees of freedom. An alternative approach is high statistics and high precision measurements which are sensitive to tiny effects that have escaped detection so far. Such effects could originate from the existence of a hidden sector of particles $[1,2]$, interacting through a messenger with the visible ones. This scenario is appealing because it provides an explanation for the excess of positrons in cosmic rays observed by PAMELA in 2008 [3] and recently confirmed by FERMI [4] and AMS [5], namely, that they are from the annihilation of dark matter particles. The lack of excess of antiprotons [6] suggests that the mass of the messenger should be below $1 \mathrm{GeV}$ or that it interacts mainly with leptons. In addition, this messenger could provide the missing contribution to the present three sigma discrepancy between experiment and theory in the muon anomalous magnetic moment $a_{\mu}=\left(g_{\mu}-2\right) / 2$ [7].

The simplest hidden sector model just introduces one extra $U(1)$ gauge symmetry and a corresponding gauge boson: the "dark photon" or $U$ boson. As in QED, this will generate interactions of the types

$$
\mathscr{L} \sim g^{\prime} q_{f} \bar{\psi}_{f} \gamma^{\mu} \psi_{f} U_{\mu}^{\prime},
$$

where $g^{\prime}$ is the universal coupling constant of the new interaction and $q_{f}$ are the corresponding charges of the interacting fermions. Not all the Standard Model particles need to be charged under this new $U(1)$ symmetry thus leading in general to a different (and sometimes vanishing) interaction strength for quarks and leptons. In the case of zero $U(1)$ charge of the quarks [8], the new gauge boson cannot be directly produced in hadron collisions or meson decays.

The coupling constant and the charges can result from a direct interaction between the Standard Model fermions and the new gauge fields or can be generated effectively through the so called kinetic mixing mechanism between the QED and the new $U(1)$ gauge bosons $[1,2]$. In the latter case the charges $q_{f}$ in (1) will be just proportional to the electric charge 


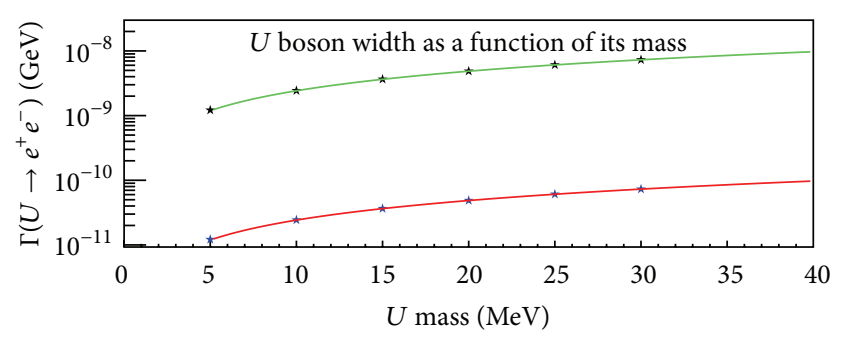

* CalcHEP, $\epsilon=0.001 \quad$ Analytic, $\epsilon=0.001$

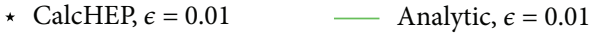

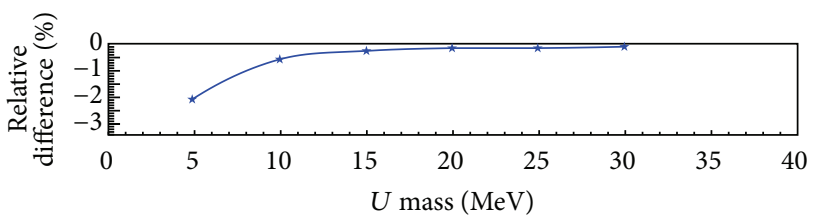

FIGURE 1: $U$ boson width calculated with formula (3) and compared to the toy model implemented in CalcHEP. Matching between the different calculations is better than $2.5 \%$.

and the associated mixing term in the QED Lagrangian will be

$$
\mathscr{L}_{\text {mix }}=-\frac{\epsilon}{2} F_{\mu \nu}^{\mathrm{QED}} F_{\mathrm{dark}}^{\mu \nu}
$$

The associated mixing coupling constant, $\epsilon$, can be so small ( $<$ $10^{-3}$ ) as to preclude the discovery of the dark photon in most of the experiments carried out so far. Another possibility is mass mixing with the $Z$, in which case the particle could also have $Z$-like properties.

In the hypothesis that the dark sector does not contain any particle lighter than the $U$ boson, and that $M_{U}$ is smaller than twice the muon mass, the $U$ can only decay to $e^{+} e^{-}$pairs. In this case the $U$ is expected to be a very narrow resonance with a total decay width given by

$$
\Gamma_{U}=\Gamma_{U \rightarrow e+e^{-}}=\frac{1}{3} \alpha \epsilon^{2} M_{U} \sqrt{1-\frac{4 m_{e}^{2}}{M_{U}^{2}}}\left(1+\frac{2 m_{e}^{2}}{M_{U}^{2}}\right),
$$

which leads to a lifetime $\tau_{U}$ proportional to $1 /\left(\epsilon^{2} M_{U}\right)$.

The present paper describes a proposal devoted to the search for such a particle in a positron on target experiment. To be able to simulate the production of the $U$ boson, the Lagrangian term from formula (1), assuming $\psi=e$, was implemented in the CalcHEP $[9,10]$ simulation software with $g^{\prime} q_{f}=\epsilon$. The results obtained for the decay width $\Gamma_{U \rightarrow e+e_{-}}$as a function of the $U$ boson mass were compared with the analytic formula (3) and are shown in Figure 1 for two different values of $\epsilon$. The difference between analytic and simulated values is less than $2.5 \%$, validating the usage of CalcHEP for the calculation of the cross sections of other processes involving the $U$ boson. In this notation the ratio of the strengths of the electromagnetic $(\alpha)$ and the new $U(1)$ $\left(\alpha^{\prime}\right)$ interactions is $\alpha^{\prime} / \alpha=\epsilon^{2}$.

\section{Present Status of Experimental Searches}

Many searches have been performed in recent years to detect low energy exotic particles like the $U$ boson exploiting different techniques that can be divided into three groups:

(i) direct searches in beam dump experiments,

(ii) fixed target experiments,

(iii) direct searches in decays of mesons like $\pi^{0}, \eta, \phi, \Upsilon$.

Based on the final state the experiment is looking at, the searches can also be divided into visible and invisible. Visible searches implement a full reconstruction of the $U$ boson decay products, usually $e^{+} e^{-}$or $\mu^{+} \mu^{-}$, and are thus less demanding in terms of the definition of the initial-state kinematics. The invisible searches do not assume that the $U$ boson decay products, if any, are detectable by the experiment. However, if there are no Standard Model particles in the final state, a more stringent limit could be obtained through more efficient background vetoing. Both types of searches are complementary and are equally important.

The limits on $U$ boson searches from beam dump experiments come from reanalysis [11] of a compete set of experiments performed at SLAC and Fermilab during the ' 80 s and ' 90 s to search for light Axion-like particles [12-14]. These studies were triggered by the observation of a $1.8 \mathrm{MeV}$ mono-energetic positron peak in heavy-ion collisions at GSI in 1983 [15]. In these experiments, $U$ bosons or axion like particles are produced, with a process similar to bremsstrahlung of ordinary photons, by a very intense electron beam incident on a dump. The produced particles travel through the dump due to their long lifetime and are observed by a detector behind the dump through their decays into $e^{+} e^{-}$pairs. In this kind of experiment, the measured quantity is in fact $\epsilon^{2} \cdot \operatorname{BR}\left(U \rightarrow e^{+} e^{-}\right)$. If in the dark sector no particle lighter than the electron exists the limits are valid; otherwise the $U$ boson will decay into this new particle and may remain undetected in the experiment. In Figure 2 the present limits for dump experiments are shown in grey in the leftmost part.

Fixed target experiments share the same beam type and production mechanism as dump experiments. The $e^{+} e^{-}$ invariant mass spectrum is searched for narrow resonances. The thickness of the target is reduced in order to allow short living $U$ boson to escape. The angular coverage of the spectrometer limits the acceptance and the accessible mass region. The exclusions from MAMI [16] and APEX [17] experiments are shown in Figure 2.

Collider experiments have searched for resonances in the $e^{+} e^{-}\left(\mu^{+} \mu^{-}\right)$mass spectra $[18,19]$ in the decay products of mesons like $\pi^{0}, \eta, \phi$, or $\Upsilon$. Searches of this kind have been performed by WASA-at-COSY [20], KLOE [21, 22], and BaBar [23]. They are limited in mass reach by the mass of the meson, they assume that the $U$ boson couples to quarks and to leptons with the same strength, and it decays into lepton pairs. The results from collider experiments are shown in the top right part of Figure 2. 


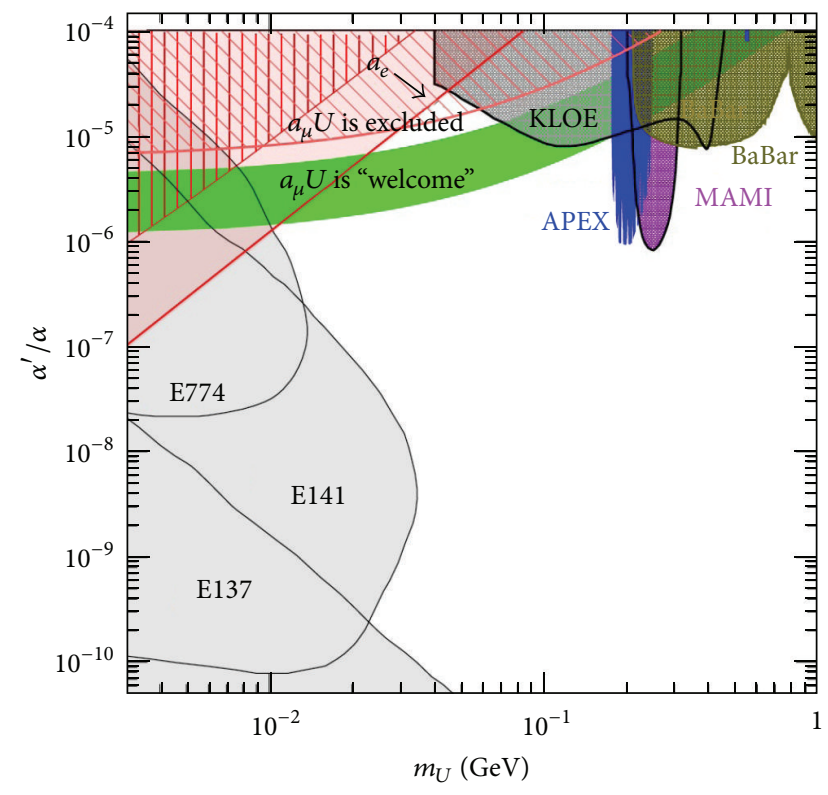

FIGURE 2: Exclusion region in the hypothesis of decay into lepton pairs.

The $U$ boson would also contribute to the anomalous magnetic moments of the electron and muon. The contribution can be written as [24]

$$
a=\frac{g-2}{2} ; \quad \Delta a=\frac{\alpha \epsilon^{2}}{2 \pi} \times f
$$

where $\alpha$ is the fine structure constant, $f=1$ for $m_{l} \gg$ $M_{U}$ and $f=2 m_{l}^{2} /\left(3 M_{U}^{2}\right)$ for $m_{l} \ll M_{U}$. At present, $\alpha$ is in fact determined from the measurement of the electron magnetic moment $g_{e}$ [25] and then is used to calculate the muon anomalous magnetic moment. Comparison between the theoretical and the experimental value allows a limit to be set in the $M_{U}-\epsilon^{2}$ parameter space, shown with diagonal stripes in Figure 2. On the contrary the well known $3 \sigma$ discrepancy between the measured value of $g_{\mu}$ and the Standard Model prediction [7] can be explained by the existence of the $U$ boson. In this hypothesis a band of mass coupling values, in green in Figure 2, is identified. This region is of particular interest and is not thoroughly explored at present.

An external knowledge of $\alpha$ is necessary to exploit the measurement of $g_{e}$ to set limits on the $U$ boson parameters. The fine structure constant was extracted from a recent single measurement of the ratio between the Planck constant and the mass of the ${ }^{87} \mathrm{Rb}$ atom [26]. The precision achieved is an order of magnitude better than that for the previous measurements, which had been conducted two years earlier. That measurement, combined with (4) and the latest tenthorder calculations of the QED contributions [27] to $g_{e}$, gives a bound, shown by the pink line in Figure 2, reaching $\epsilon^{2}<10^{-7}$ for $M_{U}=1 \mathrm{MeV}$ and $\epsilon^{2}<10^{-4}$ for $M_{U}=100 \mathrm{MeV}$. The limits from the measurements before [26] are also shown in vertical stripes.

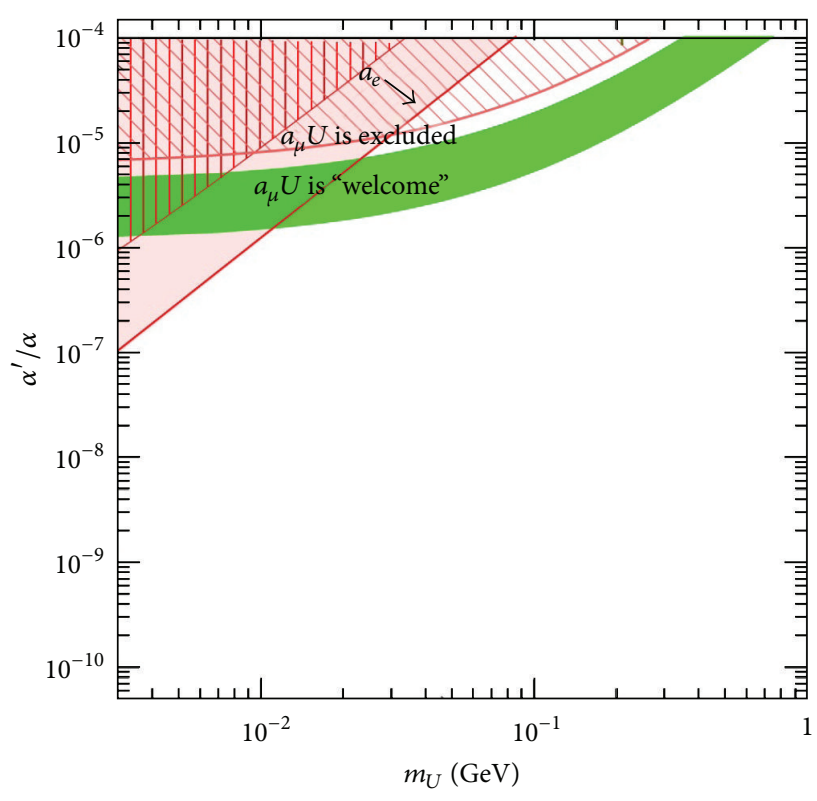

Figure 3: Exclusion region in the hypothesis of invisible $U$ boson, coupling to leptons.

There are few studies on the searches of a $U$ boson not decaying into Standard Model particles. The data by SLAC Millicharge Experiment were interpreted within this scenario [28]. The $U$ boson was assumed to decay into $\chi \chi$ and the $\chi$ to be detected by elastic scattering on atomic nuclei (through a virtual $U$ boson exchange). The exclusion obtained however depends additionally on the $\chi$ mass and the coupling between the $U$ boson and the dark matter. The $U$ boson decaying into any invisible final state can be constraint also from the branching fraction of the decays $K^{+} \rightarrow \pi^{+} \nu \bar{\nu}[24,29]$, assuming nonzero coupling to quarks. The present limits are in the range of $M_{U} \sim 100 \mathrm{MeV}$ and $\epsilon^{2} \sim 10^{-6}$. Further improvements will be possible with the upcoming NA62 results [30].

The only limits on the $M_{U}$ and $\epsilon$ below $1 \mathrm{GeV}$ that do not assume coupling to quarks and are not dependent on the possible $U$ boson decay channel are from the previously described magnetic moment measurements. This scenario, being the most general one, is illustrated in Figure 3. Since the region preferred by the present $\left(g_{\mu}-2\right)$ results is not completely covered by direct searches even for visible decays, new experiments devoted to the the search for $U$ boson are highly desirable. In addition, most of the searches of the invisible $U$ boson decays could be done with relatively small scale experiments [31].

\section{Beam Test Facility at LNF}

The DAФNE beam-test facility (BTF), shown in Figure 4, is a beam transfer-line [32] from the DAФNE linac to an independent experimental hall with a dedicated control room. It is capable of providing up to 50 bunches per second of electrons or positrons with $800 / 550 \mathrm{MeV}$ maximum energy. The minimum energy is below $250 \mathrm{MeV}$ in both electron and 


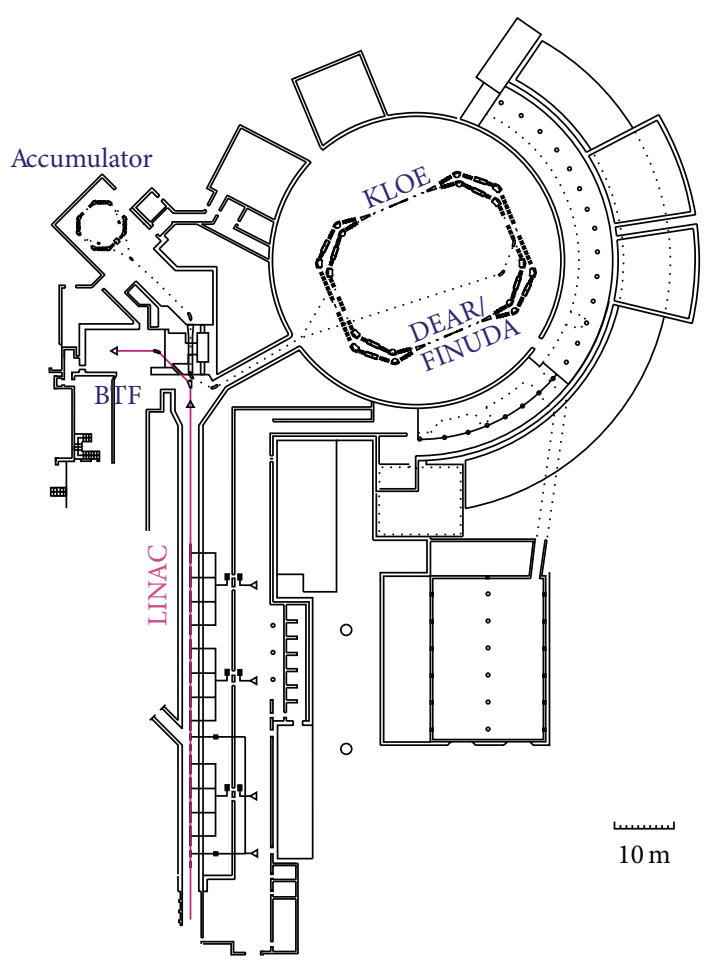

FIGURE 4: Layout of the DAФNE complex at Laboratori Nazionali di Frascati, INFN.

positron modes, even though some optimization is necessary in order to obtain stable operation. An energy upgrade of the linac is foreseen within the next three years [33], bringing the maximum energy for electrons/positrons to about $1050 / 800 \mathrm{MeV}$. Each bunch consists of microbunches with total length of 350 ps with 140 ps flat top. Beam granularity together with the subdetectors time resolution can be used to reduce the pile-up background. The typical emittance of the electron/positron beam is of $1(1.5) \mathrm{mm} * \mathrm{mrad}$, while the maximum beam current is of $500 \mathrm{~mA}(100 \mathrm{~mA})$. Beam characteristics (spot size, divergence, and momentum resolution) strongly depend on the beam energy and the number of particles per bunch. The parameters are summarized in Table 1.

A pulsed magnet allows individual bunches to be extracted into the BTF beam line. The BTF beam can be attenuated by a variable-depth copper target and energy selected with an accuracy of better than $1 \%$ before being transported to the experimental hall. In the experimental hall the particles can be either delivered on a straight beam line, or deflected by 45 degrees by a dipole magnet. In normal operation, one of the 50 pulses/second is sent to a magnetic spectrometer for a precise measurement of the beam momentum. Presently, the maximum beam intensity in the BTF experimental hall is limited by radio-protection safety regulations at $3.12 \cdot 10^{10}$ particles/second.

The most important limitation of the linac for the project described in this paper was the very short duration of the bunch, $10 \mathrm{~ns}$, and the 50 bunches/s delivered. In fact, to obtain a sufficiently high event rate high number of $e^{+}$per bunch has to be used, increasing the pile up probability. This pileup could be partially resolved by the subdetectors with a time resolution of the order of a nanosecond, but this required an increased bunch length. A recent upgrade of the pulser of the linac gun [34] now allows variation of the pulse duration between 2 and $40 \mathrm{~ns}$, but there is an indication that the bunch width can be extended up to about $200 \mathrm{~ns}$. The bunch rate, however, can not be increased at present.

The present proposal assumes that the BTF will be able to provide 50 bunches per second with duration of $40 \mathrm{~ns}$ and $10^{4}-10^{5}$ positrons.

\section{Experimental Technique}

The experiment aims to search for the production of a $U$ boson in the process

$$
e^{+} e^{-} \longrightarrow U \gamma
$$

where the positrons are the beam particles and $e^{-}$are the electrons in the target. The accompanying photon is then detected by a calorimeter regardless of the $U$ decay products. A single kinematic variable characterizing the process, the missing mass, is computed using the formula:

$$
M_{\text {miss }}^{2}=\left(P_{e^{-}}+P_{\text {beam }}-P_{\gamma}\right)^{2} .
$$

Its distribution should peak at $M_{U}^{2}$ for $U$ boson decays, at zero for the concurrent $e^{+} e^{-} \rightarrow \gamma \gamma$ process, and should be smooth for the remaining background. The approach described provides sensitivity for both visible and invisible searches.

The proposed experimental setup uses the BTF positron beam impinging on a thin target and is composed of the following parts:

(i) active target, to measure the average position of the beam during a single BTF spill,

(ii) spectrometer, to measure the charged interaction products in a given momentum range,

(iii) dipole magnet, to deflect the primary positron beam out of the spectrometer and calorimeter and to allow momentum analysis,

(iv) vacuum chamber, to minimize the unwanted interactions of primary and secondary particles,

(v) electromagnetic calorimeter, to measure and/or veto final state photons.

A schematic view of the experimental apparatus is shown in Figure 5.

The primary beam crosses the target and if a beam particle does not interact it is bent by the magnet in between the end of the spectrometer and the calorimeter, thus leaving the experiment undetected. If any kind of interaction causes the positron to lose more than $50 \mathrm{MeV}$ of energy the magnet bends it into the spectrometer acceptance, providing the veto capabilities against background. If a $U$ boson decays into $e^{+} e^{-}$, the tracks are also detected by the spectrometer. This could be used to perform visible decay searches. 


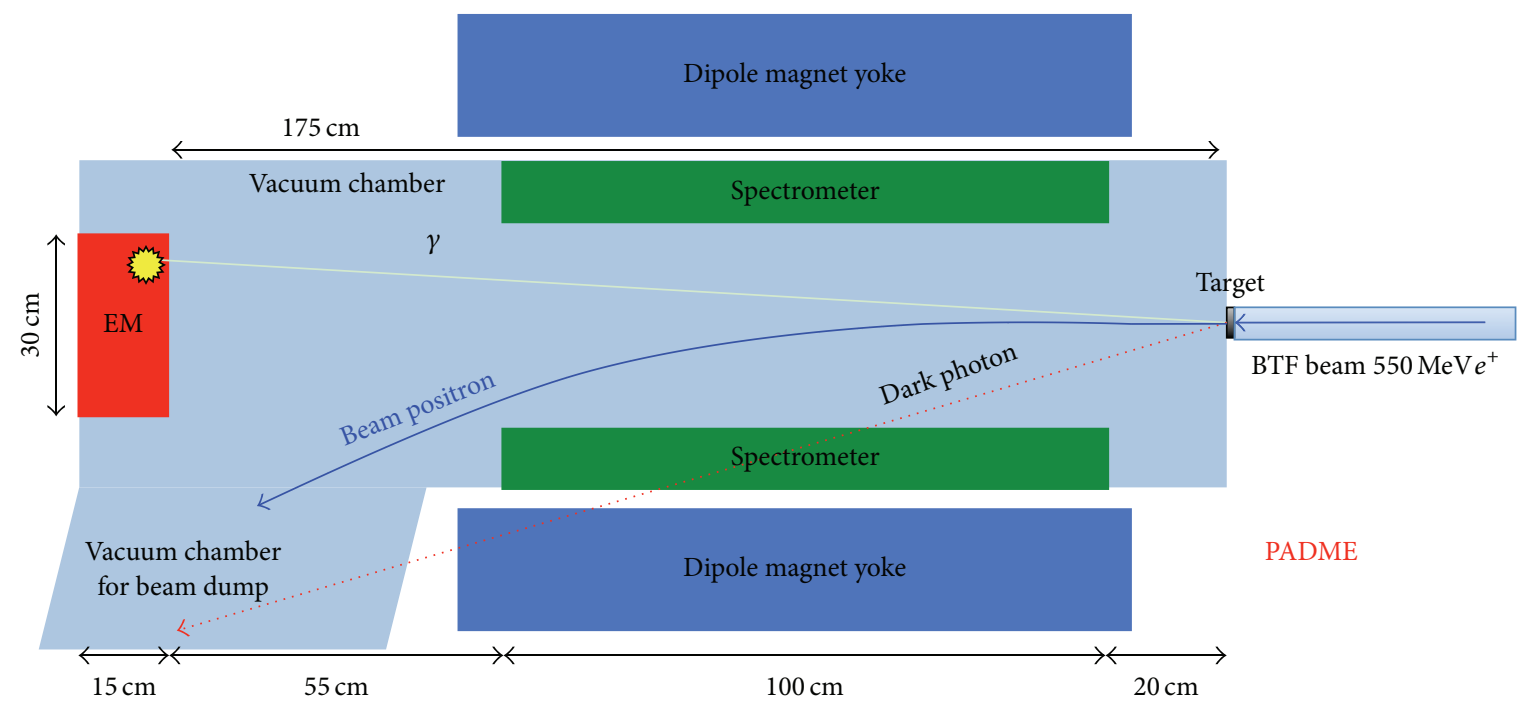

FIgUre 5: Schematic of the Positron Annihilation into Dark Matter Experiment (PADME).

TABLE 1: BTF beam parameters [32]. A possible increase of the performance after an upgrade is also shown.

\begin{tabular}{|c|c|c|c|c|}
\hline & $e^{+}$ & $e^{-}$ & $e_{\mathrm{Upg}}^{+}$ & $e_{\mathrm{Upg}}^{-}$ \\
\hline Maximal beam energy $[\mathrm{MeV}]$ & 550 & 800 & 800 & 1050 \\
\hline Beam rate [particles/bunch] & $6.2 \cdot 10^{8}$ & $6.2 \cdot 10^{8}$ & $6 \cdot 10^{9}$ & $3.1 \cdot 10^{10}$ \\
\hline Number of bunches per second $[\mathrm{Hz}]$ & 50 & 50 & 50 & 50 \\
\hline Max. averaged current during a bunch $[\mathrm{mA}]$ & 10 & 10 & 100 & 500 \\
\hline Typical emittance $[\mathrm{mm} *$ mrad] & 1.5 & 1. & 1.5 & 1. \\
\hline Beam spot size $(\sigma)[\mathrm{mm}]$ & 2. & 2. & 2. & 2. \\
\hline Bunch length [ns] & 10 & 10 & 40 & 40 \\
\hline
\end{tabular}

Due to the very thin target the majority of beam does not interact. It is transported in vacuum to the end of the experimental setup and can be reused for detector testing, provided that appropriate beam transport system is built.

The proposed experiment is compatible with the operation of the DAФNE ring. However due to the $40 \%$ reduction of the available beam time due to injection into the DAФNE machine a longer data taking period or a reduction of the sensitivity should be foreseen. A standalone operation has the advantage of profiting from beam energy variation and nominal running conditions.

4.1. $U$ Boson Production at BTF. The possible $U$ boson production mechanisms accessible in $e^{+}$-on-target collisions are $e^{+} e^{-} \rightarrow U \gamma$ and $e^{+} A \rightarrow e^{+} A U$, the so called annihilation and $U$-strahlung production.

Both processes are similar to the ones for ordinary photons, as shown in Figure 6, and their cross section scales with $\epsilon^{2}$. The present linac maximum positron energy of $550 \mathrm{MeV}$ allows the production of $U$ bosons through annihilation up to a centre of mass energy of $23.7 \mathrm{MeV}$. The kinematical constraints of the initial state are of great importance for rejecting the background.
The cross sections for annihilation and bremsstrahlung emission of an ordinary photon with energy above $1 \mathrm{MeV}$ for positrons on a carbon target are shown in Figure 7. The annihilation cross section was calculated directly with CalcHEP and was compared with the Heitler formula implemented in GEANT4 [35]. Agreement within 2\% is observed. For the bremsstrahlung the GEANT4 parametrization of the cross section was used.

Since the ratio of annihilation to bremsstrahlung cross sections is proportional to $1 / Z$, the lower the $Z$ of the target the better. However due to the requirement to form a rigid and self-supporting structure, carbon was chosen as a material, providing annihilation/bremsstrahlung ratio of $2.3 * 10^{-3}\left(5.1 * 10^{-3}\right)$ for $550 \mathrm{MeV}(250 \mathrm{MeV})$ positrons. The cross section for annihilation of positrons with energies of $550 \mathrm{MeV}(250 \mathrm{MeV})$ is $1.5 \mathrm{mb}(3 \mathrm{mb})$ per free electron leading to a probability of $6 * 10^{-6}\left(1.2 * 10^{-5}\right)$ in a $50 \mu \mathrm{m}$ thick carbon target.

Operating the beam line in the regime of $10^{4}-10^{5} e^{+}$per bunch and $50 \mathrm{bunch} / \mathrm{s}$ in one year of data taking with $60 \%$ efficiency, a sample of $\sim 10^{13}-10^{14}$ positrons on target can be collected, corresponding to $\sim\left(6 \cdot 10^{7}-6 \cdot 10^{8}\right)$ annihilation per year. In a zero background experiment, a limit down to $10^{-8}-10^{-9}$ in $\epsilon^{2}$ could be set. 


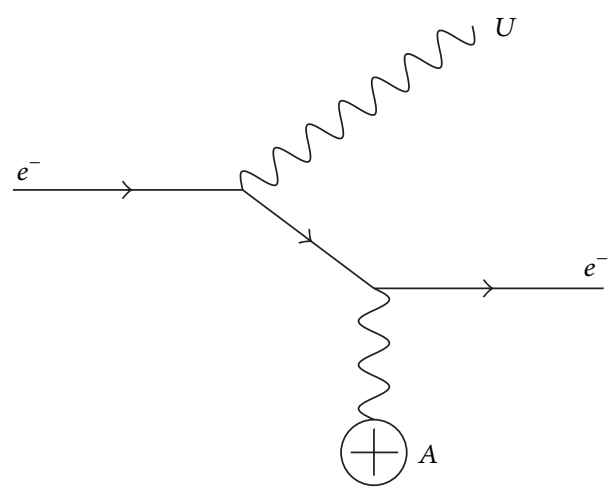

$U$-strahlung

(a)

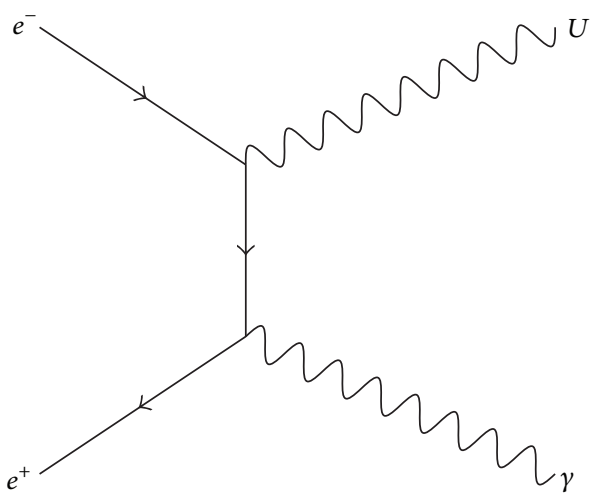

Annihilation

(b)

FIgURE 6: $U$ boson production mechanisms.

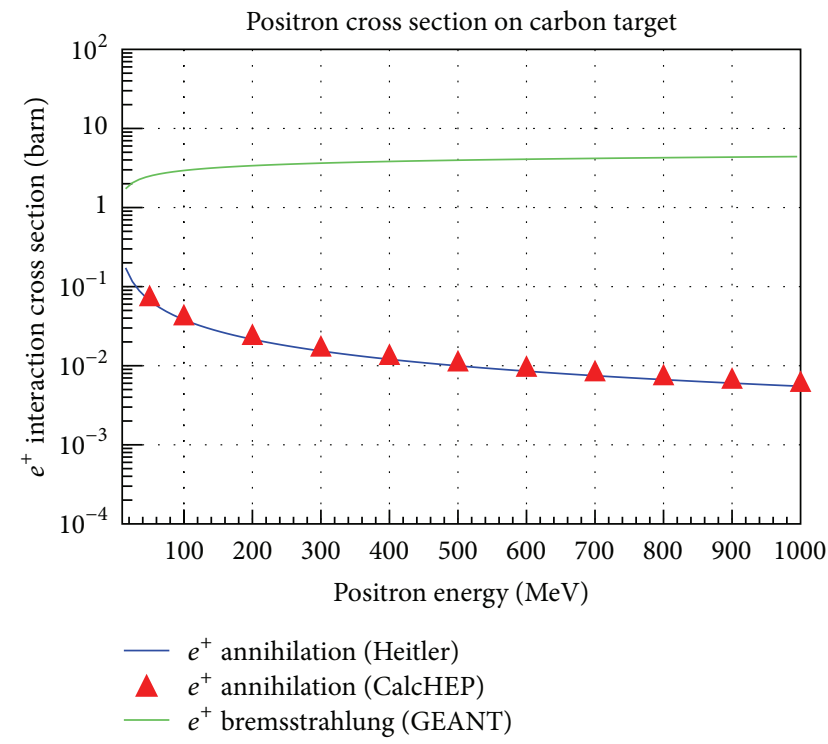

FIgURE 7: Positron cross section on a carbon target.

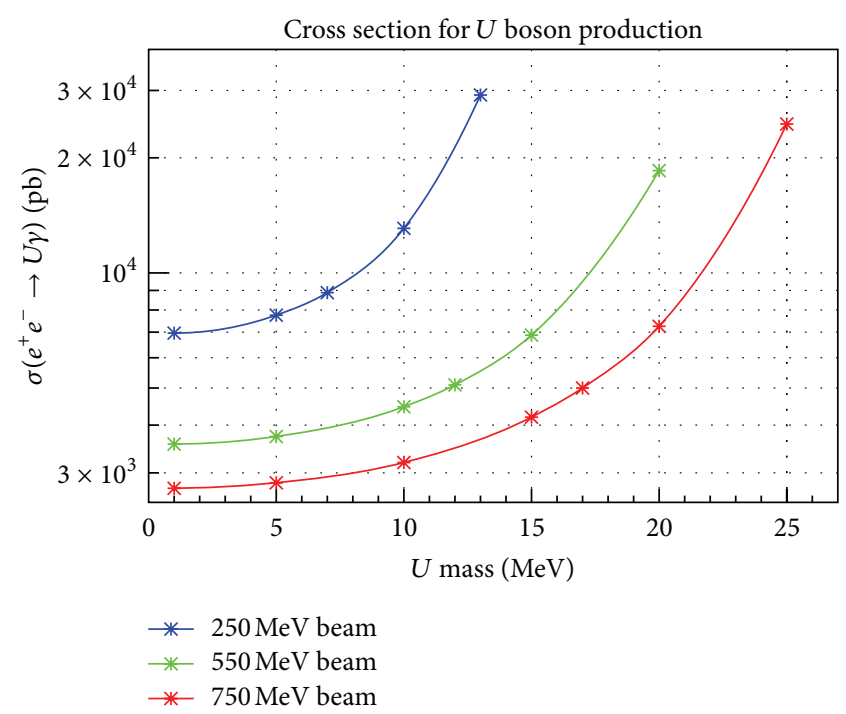

Figure 8: $U$ boson production cross section (for $\epsilon=10^{-3}$ ) as a function of mass for different beam energies.

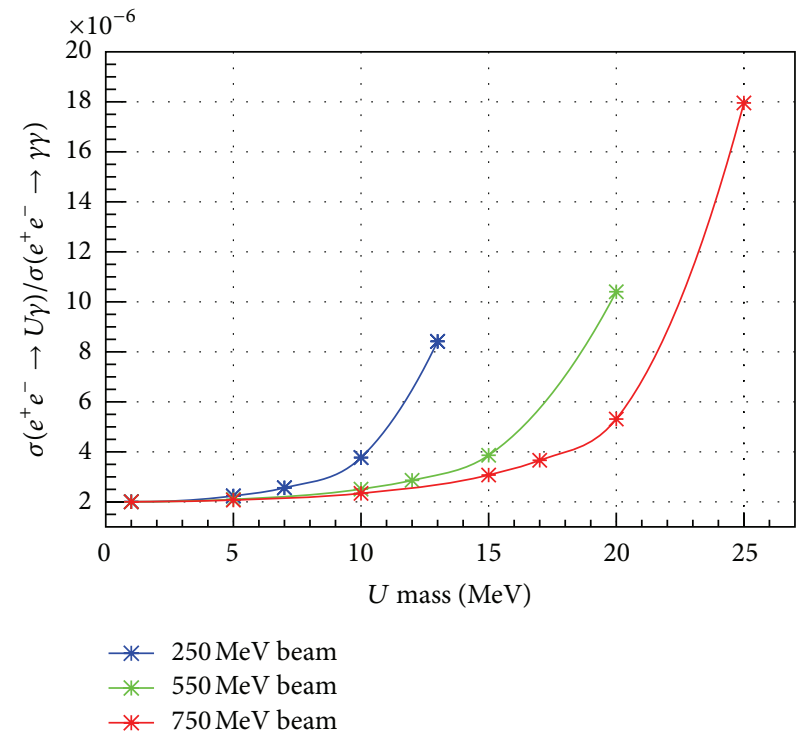

FIGURE 9: Ratio of the $U$ boson production (for $\epsilon=10^{-3}$ ) and twophoton annihilation cross section as a function of the $U$ boson mass for different beam energies.

Another advantage of the $U$-annihilation production process comes from the resonant enhancement of the $U$ boson production cross section. The production cross section, shown for different beam energies in Figure 8, and the $\sigma(U \gamma) / \sigma(\gamma \gamma)$, shown in Figure 9, rise fast when the mass of the $U$ boson approaches the available CM energy. The ratio of the the annihilation to bremsstrahlung cross section also increases with the decrease of beam energy (as seen in Figure 7) giving an additional rise to the production of the $U$ boson. This can be exploited by an experiment at the the BTF beam line due to its variable beam energy and could enhance the sensitivity to low-mass $U$ bosons. 


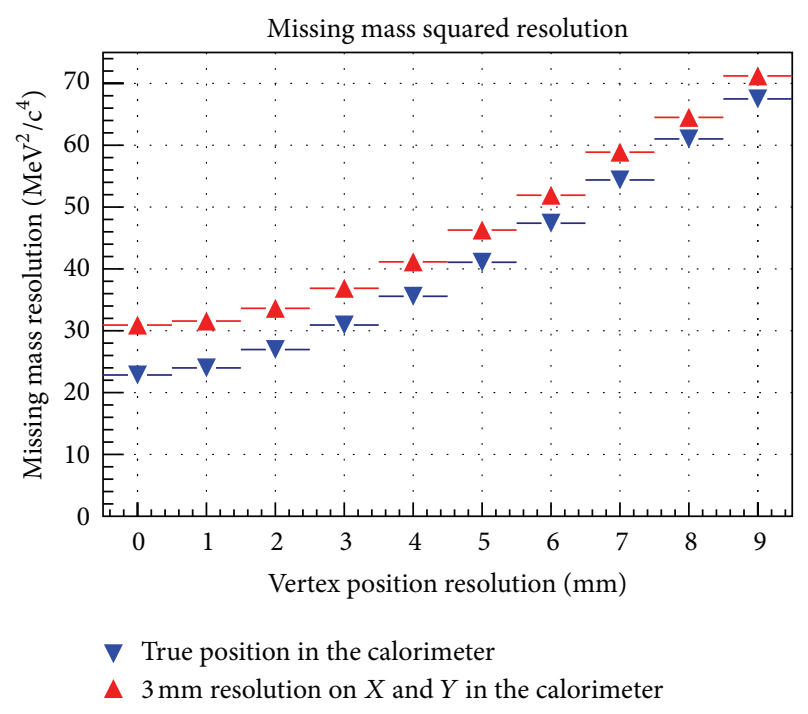

FIGURE 10: Dependence of the missing mass squared resolution on the vertex position resolution for a $U$ boson with $M_{U}=15 \mathrm{MeV}$.

4.2. Active Target. Scattering inside the target material worsens the knowledge of the momentum and direction of the primary beam and broadens the reconstructed missing mass spectrum. In fact, in the invisible searches the kinematics cannot be closed without assumptions on the decay vertex and beam direction, while in the visible searches, the vertex position helps in rejecting fake tracks. For this reason the usage of $50 \mu \mathrm{m}$ target is proposed, for which the simulation showed that the relation $E_{\gamma \text {,brems }}+E_{e+}=E_{\text {Beam }}$ is fulfilled with a resolution better than the initial beam spread (1\%). In addition, the probability of a single annihilation is $\sim 5 \%$ per bunch with $\sim 10^{4}$ positrons.

The importance of the beam position measurement can be seen in Figure 10, where the missing mass resolution for a $U$ boson with mass $15 \mathrm{MeV}$, assuming perfect calorimeter positioning and a realistic $3 \mathrm{~mm}$ resolution on the cluster position inside the calorimeter, is shown. In order to obtain missing mass resolution down to $\sim 30 \mathrm{MeV}^{2} / \mathrm{c}^{4}$ it is necessary to provide better than $2 \mathrm{~mm}$ position resolution on the beam spot on the target, which is used for the calculation of the angle of the emitted photon. A single BTF bunch features such a small spot but it is difficult to achieve long term positioning stability due to the instability of the currents in the bending magnets [36].

The design of the proposed target, shown schematically in Figure 11, consists of two orthogonal layers of ten strips made of carbon, each with dimensions $2 \mathrm{~mm} \times 50 \mathrm{~mm} \times 25 \mu \mathrm{m}$, mounted on a vacuum flange. Both diamond and graphite could possibly be used due to their tolerance to radiation damage and good heat conductivity. The latter is necessary to avoid having to use a cooling system while operating in high intensity beams.

The target will also act as a beam monitor detector, providing the beam spot position bunch per bunch. Its readout will be based on measurement of the secondary electron emission (graphite) or Cherenkov light (diamond).

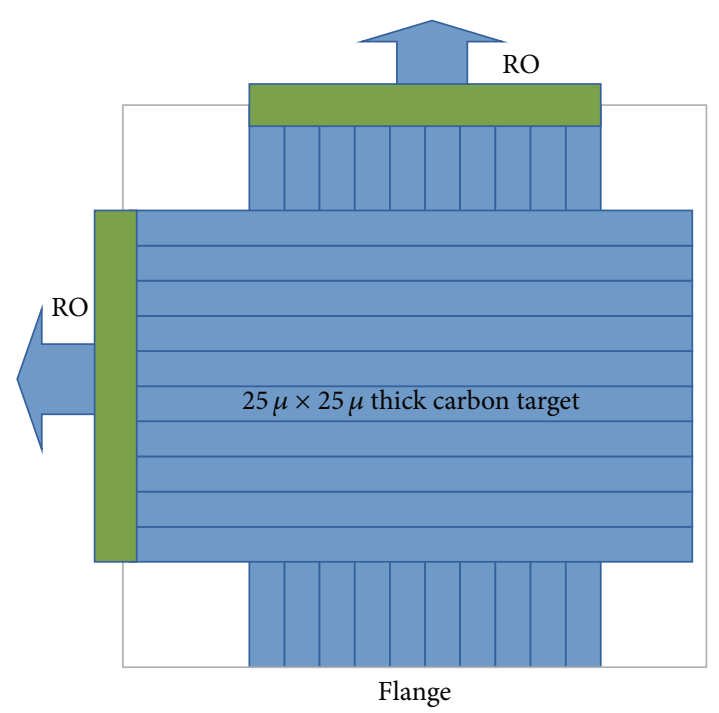

FIGURE 11: Schematics of the beam monitor. A design with $2 \mathrm{~mm}$ strips both in horizontal and in vertical direction is foreseen.

By reconstruction of the beam spot, the uncertainty on the beam geometry will be reduced to that of only a single bunch. An online, two-dimensional beam image would also be useful to optimize the beam parameters while setting up the beam. In addition, it can also be used to measure the number of particles per bunch for intensities of $<10^{5}$ positron/bunch, at which methods based on induced current start to be ineffective.

4.3. The Dipole Magnet. After crossing the active target the charged remnants of the primary beam will be swept away by a sweeping magnet. Because the target is very thin, most of the beam particles will remain in the beam, in the worst case with slightly degraded energy. Due to the low energy of the beam and relaxed spatial constraints, the magnet can be a conventional one. Geant 4 simulations show that a magnetic field of $\sim 0.6$ Tesla is enough to deflect the primary $550 \mathrm{MeV}$ beam out of the calorimeter acceptance while keeping most of the electrons from $U$ boson decays inside of the spectrometer acceptance. The magnet surrounds the vacuum region and contains the whole tracking detector, thus serving as a spectrometer magnet. A gap of $50 \mathrm{~cm}$ between the coils and a uniform field of length $1 \mathrm{~m}$ is assumed.

4.4. The Spectrometer. The spectrometer role is to suppress by a factor of 100 the background from bremsstrahlung events by detecting the primary beam positrons. A tracking detector inside the magnetic field will be used to detect charged particles and to measure their momentum. The spectrometer could be either a cylindrical chamber with $20 \mathrm{~cm}$ inner radius and $25 \mathrm{~cm}$ outer radius or composed by two planar trackers, one on each of the positron and the electron deflection sides. It is expected to measure the coordinates of a crossing track with a precision of $300 \mu \mathrm{m}$ in each layer. The size of the spectrometer is defined by the condition that it should detect charged particles with momentum from $50 \mathrm{MeV}$ to $500 \mathrm{MeV}$ 
TABLE 2: Main parameters of the LYSO (Ce) crystals.

\begin{tabular}{lcccccc}
\hline Density $\left(\mathrm{g} / \mathrm{cm}^{3}\right)$ & $X_{0}(\mathrm{~cm})$ & $R_{M}(\mathrm{~cm})$ & $\tau(\mathrm{ns})$ & $\lambda_{\text {peak }}(\mathrm{nm})$ & L.Y. & $d Y / d T(\% / \mathrm{C})$ \\
\hline 7.40 & 1.14 & 2.07 & 40 & 402 & $85 \%$ & \\
\hline
\end{tabular}

travelling along the beam axis with an efficiency of $99 \%$. Positron with momentum $\vec{p}=550 \mathrm{MeV}$ should be deflected outside its acceptance. In order to avoid conversions of the emitted bremsstrahlung photons the tracker should be at least $20 \mathrm{~cm}$ far from the beam line.

The proposed detector is with five active layers and a total length of $1 \mathrm{~m}$. Few technologies are considered at present. A possible solution is a triple GEM chamber if a thin vacuum pipe on the positron path does not worsen significantly the background rejection. An alternative option is a scintillating fibre tracker that could be operated in vacuum. A hybrid solution (plastic scintillator for good time resolution and a GEM tracker) is also considered.

4.5. The Decay Chamber. Due to the high intensity of the beam and the extremely thin target $\left(0.04 \% X_{0}\right), e^{+}$interactions in air can produce a significant contribution to the background. In fact, since the radiation length of air is $285 \mathrm{~m}$ at a pressure of $1013 \mathrm{mbar}$ and the distance from the calorimeter to the target is $\sim 2 \mathrm{~m}$, the atmospheric air thickness is $0.7 \% X_{0}$, which is much larger than the thickness of the target itself.

An MC simulation performed with 1 mbar pressure showed a significant increase in the background with respect to an experiment in vacuum. In interactions in the residual air, the kinematical constraints (i.e., from missing mass) are weakened since the information on the interaction position and the initial beam momentum is completely lost. In addition, the emitted bremsstrahlung photons do not travel through the central calorimeter hole. For the same reason, beam particles not interacting in the target must be transported in vacuum to the dump. Due to the fact that the spectrometer itself contains gas and will not intercept the primary beam, it can be placed outside the vacuum region. A vacuum with pressure $10^{-1}$ mbar was chosen for the experiment, which makes the background contribution from beam-air interactions negligible.

4.6. The Electromagnetic Calorimeter. The electromagnetic calorimeter is responsible for the reconstruction of the photon 4-momentum. An energy resolution of better than $\sim 5 \%$ for photons with energies down to $100 \mathrm{MeV}$ and a cluster position resolution of $3 \mathrm{~mm}$ are needed in order to achieve squared missing mass resolution of $30 \mathrm{MeV}^{2} / \mathrm{c}^{4}$. In addition, a compact shower development is desired to minimize overlapping of signal and background photon clusters.

To achieve such performance, a highly segmented, inorganic crystal calorimeter made of LYSO was chosen due to its characteristics, listed in Table 2. LYSO has high density, very short $X_{0}$, and small Moliere radius together with high light output and short decay time. The calorimeter is an approximate cylinder with a diameter of $30 \mathrm{~cm}$ and depth of $15 \mathrm{~cm}$ filled with $1 \times 1 \times 15 \mathrm{~cm}^{3}$ crystals with a round shaped central hole of $4 \mathrm{~cm}$ radius, as shown in Figure 12. The active volume will be $9840 \mathrm{~cm}^{3}$ for a total of 656 crystals. Resolutions down to

$$
\frac{\sigma_{E}}{E}=\frac{1.1 \%}{\sqrt{E}} \oplus \frac{0.4 \%}{E} \oplus 1.2 \%
$$

have been recently obtained in the $\mathrm{R} \& \mathrm{D}$ for the calorimeter of the SuperB project in tests with LYSO crystals at the BTF [36].

The high segmentation of the electromagnetic calorimeter in the plane transverse to the beam direction assures a spatial resolution of $1 / \sqrt{1} 2=3 \mathrm{~mm}$ which is equivalent to an angular resolution at $1.75 \mathrm{~m}$ from the target below $2 \mathrm{mrad}$. Energy and angular resolutions obtained with a GEANT4 simulation are in agreement with the performance described above.

The average occupancy of the calorimeter varies from $5 \%$ to about $22 \%$ for bunches from $10^{4}$ to $10^{5}$ positrons. The dependence is not linear due to the splitting of clusters in the inner calorimeter region. This is crucial characteristic since it allows efficient and safe vetoing of events with more than one reconstructed cluster.

\section{MC Simulation and Reconstruction}

To understand the actual sensitivity of the proposed experiment to $U$ bosons, a full GEANT4 simulation has been developed. The simulation describes in detail the segmentation of the calorimeter and produces energy deposits for each single crystal. The magnetic field is considered to be uniform and transverse to the beam direction. The spectrometer is modelled as an active volume from which the energy of the crossing particles is retrieved without any reconstruction. The simulation does not include any passive material and does not simulate the dumping of the primary beam.

To describe the bunch structure, a simultaneous multipositron gun was implemented, taking into account beam spot size and energy spread in each single burst. The simulation uses the GEANT4 low-energy electromagnetic libraries, including two-photon annihilation, ionization processes, Bhabha and Moller scattering, and production of $\delta$-rays. A custom generator was developed to simulate the production of the $U$ boson and its eventual decay into $e^{+} e^{-}$or invisible and the three-photon annihilation.

The physical properties (lifetime and decay kinematics) of the $U$ boson were made dependent on two external parameters, allowing the change of the acceptance for different $U$ boson decay points to be studied. A complete mass scan was performed.

A cluster reconstruction algorithm providing energy and position was implemented, starting from the energy deposits in each of the calorimeter crystals. Initially, a seed crystal 


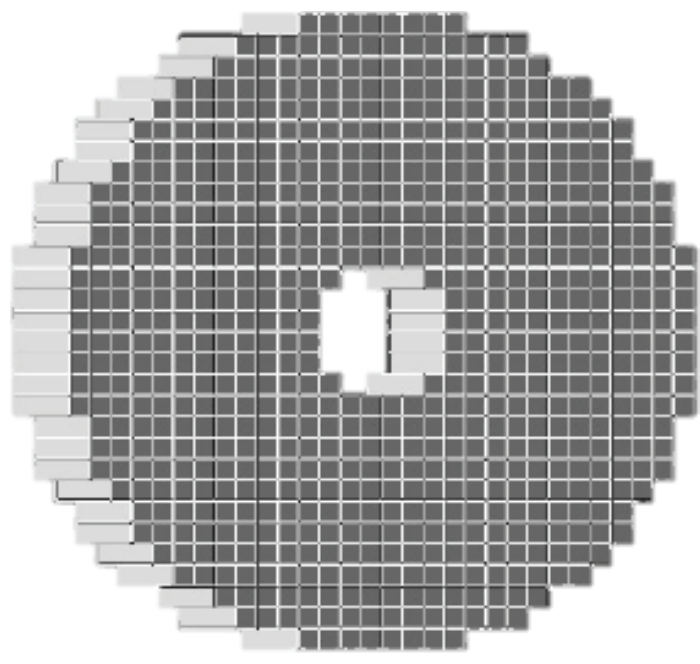

Figure 12: GEANT4 model of the EM calorimeter.

is identified, defined as the one with the maximum energy among all the cells in the calorimeter. A seed is created only if the energy is greater than $10 \mathrm{MeV}$ to reject clusters from low energy radiated photons. This condition does not introduce inefficiency in reconstructing photons from $U$ boson production in the mass region under study. The cluster is built by summing the energy of all the crystals with $E>0.1 \mathrm{MeV}$ and distance less than $4.6 \mathrm{~cm}$ from the seed, corresponding to 2.5 Moliere radii. All the cells contributing to the cluster are then excluded and the algorithm is repeated until no seed is found. The cluster position is computed by the energy weighted average of the central positions of the cells involved. Due to the relatively high number of cells involved (up to $\sim 40-50$ ) and the small crystal size a spatial resolution as low as $3 \mathrm{~mm}$ is achieved. The efficiency for cluster reconstruction is $\sim 98 \%$. The present algorithm is not very robust against overlapping clusters but is suitable for the purposes of this analysis.

\section{Analysis Strategy}

The $U$ boson coupling constant can be determined using the formula

$$
\frac{\sigma\left(e^{+} e^{-} \longrightarrow U \gamma\right)}{\sigma\left(e^{+} e^{-} \longrightarrow \gamma \gamma\right)}=\frac{N(U \gamma)}{N(\gamma \gamma)} * \frac{\operatorname{Acc}(\gamma \gamma)}{\operatorname{Acc}(U \gamma)}=\epsilon^{2} * \delta,
$$

where $N(U \gamma)=N(U \gamma)_{\text {obs }}-N(U \gamma)_{\text {bkg }}$ is the number of the signal candidates after the background subtraction, $N(\gamma \gamma)$ is the number of observed annihilation events, $\delta$ is the $e^{+} e^{-} \rightarrow U \gamma$ cross section enhancement factor, as described in Figure 9, and $\operatorname{Acc}(\gamma \gamma)$ and $\operatorname{Acc}(U \gamma)$ are the corresponding acceptances for the signal and normalization channels.

The candidates selection is based on the missing mass squared variable, calculated according to formula (6), with the target electrons assumed to be at rest $\left(\vec{P}_{e^{-}}=\overrightarrow{0}\right)$ while for the beam a nominal momentum of $550 \mathrm{MeV}$ along the $Z$ axis is used. The signal region is defined as $\pm 1.5 \sigma_{M_{\text {miss }}^{2}}$ around

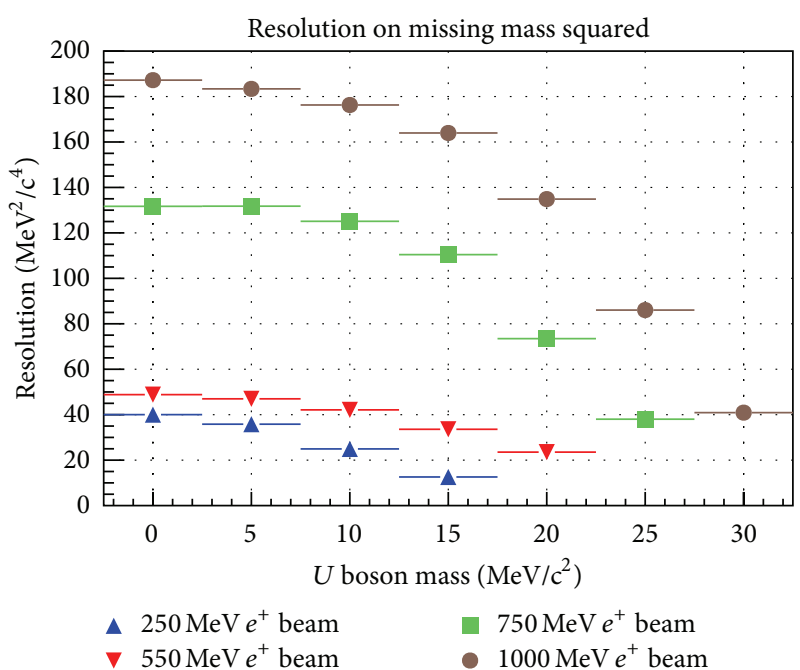

FIgURE 13: Missing mass resolution as a function of the $U$ boson mass for four different energies of the impinging positron beam.

the reconstructed value of $M_{U}^{2}$, with resolution $\sigma_{M_{\text {miss }}^{2}}\left(M_{U}\right)$ shown in Figure 13.

A simple and preliminary selection has been developed to address the possibility of performing a model independent search for a $U$-boson. It has been applied to both events with visible and invisible $U$-boson decays. Since the background estimation does not depend on the $U$ boson decay channel the only difference is the change in the acceptance for the two cases.

The selection cuts applied are the following.

(i) Only one cluster in the calorimeter. This cut rejects most of the annihilation events.

(ii) Cluster energy within $E_{\min }\left(M_{U}\right)<E_{\mathrm{Cl}}<E_{\max }\left(M_{U}\right)$. The energy cut is intended to reject low-energy photons from bremsstrahlung radiation and to define a maximum energy of the positron in the spectrometer acceptance to avoid pile-up. The energy spectrum of the recoil photons is different for different $U$ masses, resulting in $E_{\min }\left(M_{U}\right)$ varying over the interval $50-150 \mathrm{MeV}$ while $E_{\max }\left(M_{U}\right)$ is between 120 and $350 \mathrm{MeV}$.

(iii) Cluster radius in the calorimeter $5 \mathrm{~cm}<R_{\mathrm{Cl}}<13 \mathrm{~cm}$. This cut reduces the energy leakage and improves $M_{\text {miss }}^{2}$ resolution.

(iv) Positron veto in the spectrometer.

The positron veto cut aims to completely reject the bremsstrahlung gamma by detecting the beam positron inside the spectrometer. Due to the requirement of $E_{\gamma}>50 \mathrm{MeV}$, the positron is deflected out of the beam by the magnet and enters the acceptance of the tracker. The veto condition must not reject the decays of the $U$ bosons, if any. In case a positron with energy below $500 \mathrm{MeV}$ is detected, $E_{e+}+E_{\gamma}$ has to be lower than $500 \mathrm{MeV}$. In the $U$ boson decay case this cut is equivalent to the soft requirement that the electron energy is higher than $100 \mathrm{MeV}$. However if 


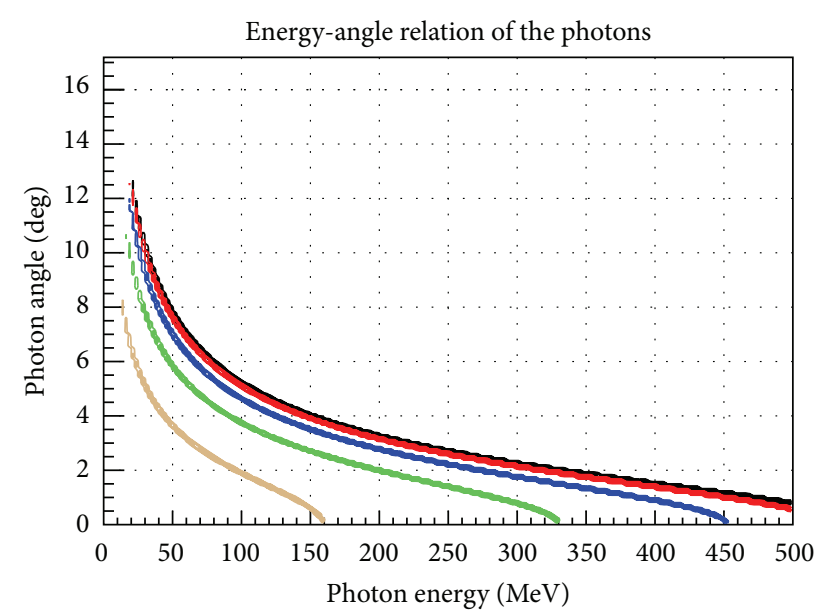

$e^{+} e^{-} \rightarrow \gamma \gamma$
$-e^{+} e^{-} \rightarrow \gamma U, M_{U}=5 \mathrm{MeV}$
$-e^{+} e^{-} \rightarrow \gamma U, M_{U}=10 \mathrm{MeV}$
$e^{+} e^{-} \rightarrow \gamma U, M_{U}=15 \mathrm{MeV}$
$e^{+} e^{-} \rightarrow \gamma U, M_{U}=20 \mathrm{MeV}$

FIGURE 14: Gamma energy versus the opening angle for $e^{+} e^{-} \rightarrow \gamma \gamma$ or $\rightarrow U \gamma$ for different $U$ boson masses. Beam energy is $550 \mathrm{MeV}$.

a high energy electron $E_{e}>200 \mathrm{MeV}$ is present the event is kept. The latter is necessary to avoid rejection of events due to pile-up.

With this selection, an acceptance of $\sim 20 \%$ was achieved for $U$ boson mass up to $20 \mathrm{MeV}$.

6.1. Background Estimation. The $U$ boson sensitivity is limited by the single-photon background. It was estimated by applying the signal selection cuts to the $e^{+}$-on-target $\mathrm{MC}$ sample and counting the number of events in the signal region.

In bremsstrahlung events, the sum of the energies of the positron track and the cluster should be equal to the beam energy since the target is very thin. In $\gamma \gamma$ events the final state has only two clusters and the sum of their energies is equal to the beam energy.

The bremsstrahlung, the process with highest cross section, leads to production of many low energy photons emitted mostly at small angles. For carbon target and $550 \mathrm{MeV}$ beam energy, about $5 \times 10^{2}$ photons with energy $>1 \mathrm{MeV}$ are produced for each annihilation interaction (see Figure 7). The calorimeter has a central hole with an aperture of 1.5 degrees minimizing the sensitivity to these photons.

Because of the closed kinematics the energy and the angle of the annihilation photons are correlated. Due to detector resolution the kinematical region allowed for annihilation photons overlaps that for a low mass $U$ boson (see Figure 14). To reduce background of this type, a veto on extra clusters in the calorimeter is applied, leading to a negligible contribution from $\gamma \gamma$ final states. The energies of photons produced by synchrotron radiation are very low, at most reaching the hard X-ray region $(\sim 10 \mathrm{KeV})$. Nevertheless the deflection

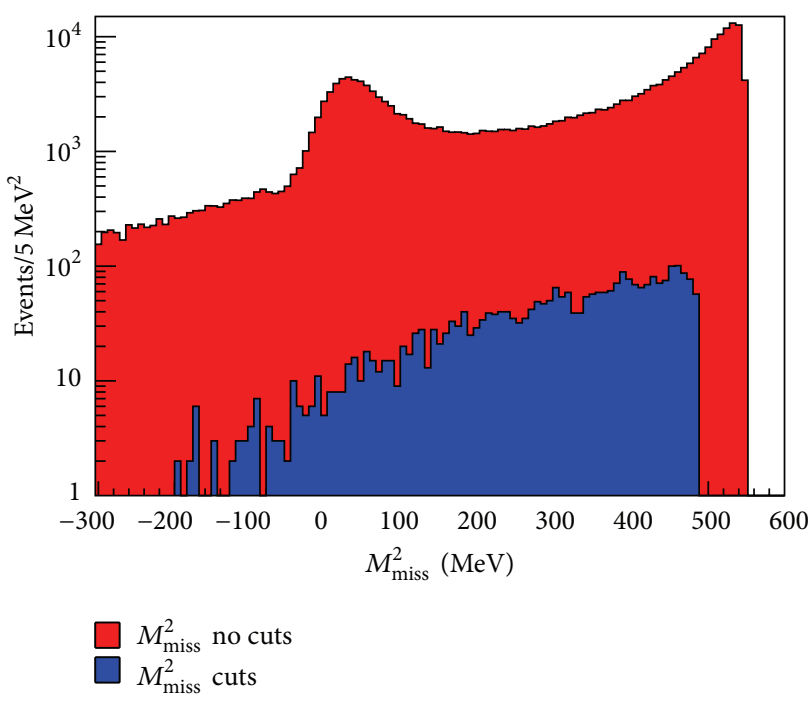

FIGURE 15: $M_{\text {miss }}^{2}$ for background events: in red with no selection cuts; in blue after all cuts applied.

of the intense beam can give rise to several thousands of these photons, slightly worsening the calorimeter energy resolution.

An additional background originates from the process $e^{+} e^{-} \rightarrow \gamma \gamma \gamma$. When just a single photon is detected by the calorimeter the reconstructed missing mass is equal to the invariant mass of the two missing photons and lies in the signal region. That scenario represents an irreducible background when the two photons fly through the inner hole of the calorimeter. Initial state radiation (ISR) with energy less than $5 \mathrm{MeV}$ decreases the visible $e^{+} e^{-}$invariant mass from $23.7 \mathrm{MeV}$ to $23.6 \mathrm{MeV}$ and is taken into account through simulating the $1 \%$ beam energy spread. The emission of a hard photon was studied using CalcHEP. The relative cross section was obtained to be $\sigma\left(e^{+} e^{-} \rightarrow \gamma \gamma \gamma\right) / \sigma\left(e^{+} e^{-} \rightarrow\right.$ $\gamma \gamma)_{E(\gamma)>5 \mathrm{MeV}}=7.5 * 10^{7} \mathrm{pb} / 1.8 * 10^{9} \mathrm{pb}=4.2 \%$ and the generated events were traced through the experimental setup to obtain the remaining background.

Other background processes like Bhabha scattering and pile-up of annihilation events are included in the background estimation through the GEANT4 simulation of the interactions of the primary positron beam. Double annihilation events produce contain extra clusters and due to energy/angle relation are additionally suppressed with respect to single ones.

Figure 15 shows the distribution of the simulated background events before (red) and after (blue) the described selection. The annihilation background peaks at $M_{\text {miss }}^{2}=0$; the bremsstrahlung is located in the region of high $M_{\text {miss }}^{2}$ values while the three-photon background populates the entire region.

6.2. Positron Flux Measurement. The total number of annihilation events $(N(\gamma \gamma) / \operatorname{Acc}(\gamma \gamma))$, which are used for normalization, can be determined in two independent ways. The first is to exploit the active target for the measurement of 
the beam flux and use the known value of $\sigma_{e^{+} e^{-} \rightarrow y \gamma}$. The signal produced by the active target is proportional to the number of positrons crossing the target itself. Its calibration can be performed by shooting the beam directly onto the calorimeter through the target, measuring the energy deposit and comparing it with the signal from the active target.

Using this curve the number of primary positrons, and therefore the total flux, can be measured in each single bunch. Then the number of annihilations can be calculated as

$$
N_{\gamma \gamma}^{\text {tot }}=\frac{N_{\gamma \gamma}}{\operatorname{Acc}_{\gamma \gamma}}=\operatorname{Flux}\left(e^{+}\right) \cdot \sigma_{\gamma \gamma},
$$

since the cross section for the annihilation process $\left(\sigma_{\gamma \gamma}\right)$ is known with very good precision (Figure 7).

Alternatively, direct reconstruction of the $e^{+} e^{-} \rightarrow \gamma \gamma$ annihilation events can be performed. For the selection of $\gamma \gamma$ events the same geometrical cuts for signal events are used. Two clusters in the calorimeter are required, each with reconstructed energy $100 \mathrm{MeV}<E_{\mathrm{Cl}}<400 \mathrm{MeV}$ and radius $5 \mathrm{~cm}<R_{\mathrm{Cl}}<13 \mathrm{~cm}$. The $\gamma \gamma$ invariant mass is reconstructed assuming the particles come from the target $Z$ position and using the formula

$$
M_{\gamma \gamma}=\frac{\sqrt{\left[\left(X_{\gamma 1}-X_{\gamma 2}\right)+\left(Y_{\gamma 1}-Y_{\gamma 2}\right)\right] E_{\gamma 2} E_{\gamma 2}}}{Z_{\text {EMcal }}-Z_{\text {Target }}} .
$$

In Figure 16 the distribution of $M_{\gamma \gamma}$ from MC is shown. The genuine $\gamma \gamma$ events (in red) peak at the centre of mass energy of the $e^{+} e^{-}$pair while the negligible bremsstrahlung background (in blue) is situated at small $M_{\gamma \gamma}$. The expected contamination from bremsstrahlung processes in the signal region is well below the $0.1 \%$ level. The resolution on $M_{\gamma \gamma}$ is found to be $1 \mathrm{MeV}$. Using the GEANT4 simulation and the invariant mass cut $20 \mathrm{MeV}<M_{\gamma \gamma}<26 \mathrm{MeV}$, the acceptance for this selection has been measured to be $\sim 7 \%$, with a calorimeter geometrical acceptance for two photons of $\sim 17 \%$. The expected precision in the measurement of the flux is dominated by the value of the annihilation cross section, since the statistical error on $\gamma \gamma$ sample is $\sim 0.05 \%$.

Combining the two results for the number of primary positrons, the cross section for the annihilation process $\left(\sigma_{\gamma \gamma}\right)$ can be measured as a by-product. This will help to cross check the reliability of the obtained flux, minimizing the systematics.

6.3. Sensitivity. With the described experimental setup and simulation technique $10^{11}$ positrons on target were generated $\left(10^{7}\right.$ events each with $10^{4}$ positron and $10^{8}$ events each with $10^{3}$ positron) in order to study the effect of pile-up events. In addition, samples of 1000 events were generated for $U$ boson masses $2.5,5,7.5,10,12.5,15,17.5$, and $20 \mathrm{MeV}$, with a single $U$ boson per bunch with $2 * 10^{3}$ positrons, corresponding to $4 * 10^{4}$ positrons per $40 \mathrm{~ns}$ bunch. This assumes that the detector is able to match clusters and tracks within $2 \mathrm{~ns}$. The missing mass distributions for some of the masses are shown in Figure 17. The acceptance (Figure 18) and the expected number of background events (Figure 19) were obtained by running the events through the selection.

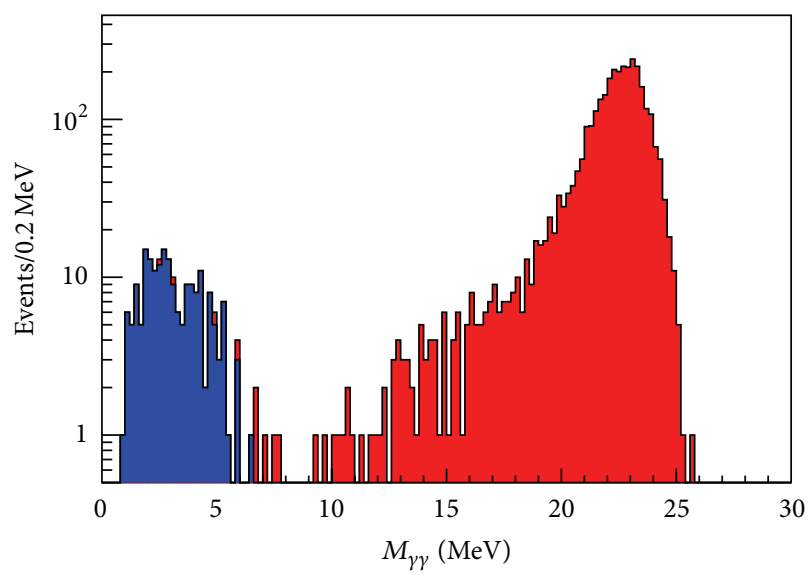

$\gamma \gamma$ mass

$\gamma \gamma$

Brems

FIGURE 16: $M_{\gamma \gamma}$ mass distribution.

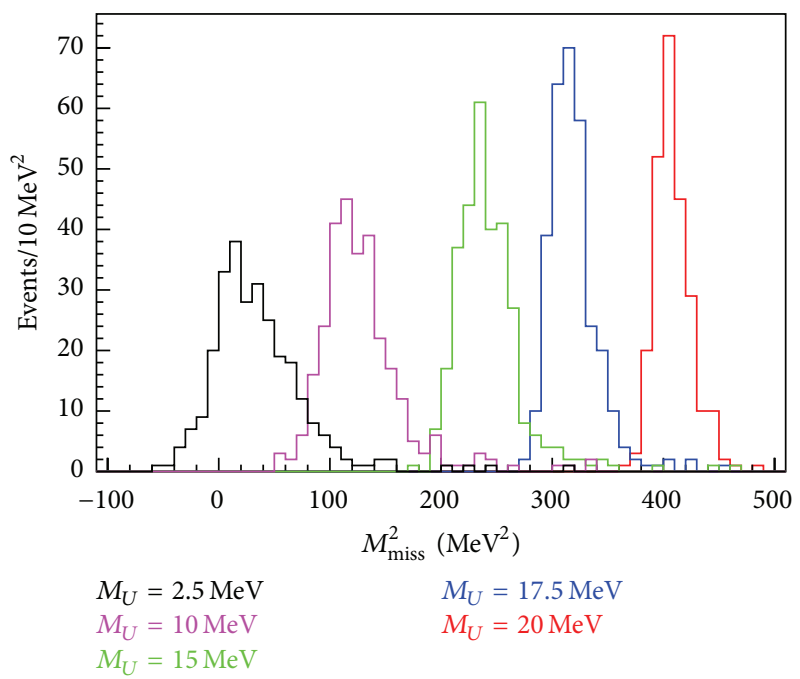

FIGURE 17: Missing mass squared distribution as a function of $U$ boson mass.

The background was further scaled by a constant factor of 400 to account for one year of running of the experiment with $60 \%$ efficiency, $4 * 10^{4}$ positrons per bunch, corresponding to a total of $4 * 10^{13}$ positrons on target. Under the assumption of no signal, an upper limit on the coupling $\epsilon$ can be set, using the statistical uncertainty on the background. The expected exclusion region is shown in Figure 20 for both the visible and the invisible channels.

\section{Conclusions and Discussion}

Searches for dark photons are well motivated by recently observed phenomena. The analysis presented in this paper shows that such a search can be performed exploiting the present linac of the DAФNE facility in just one year of 


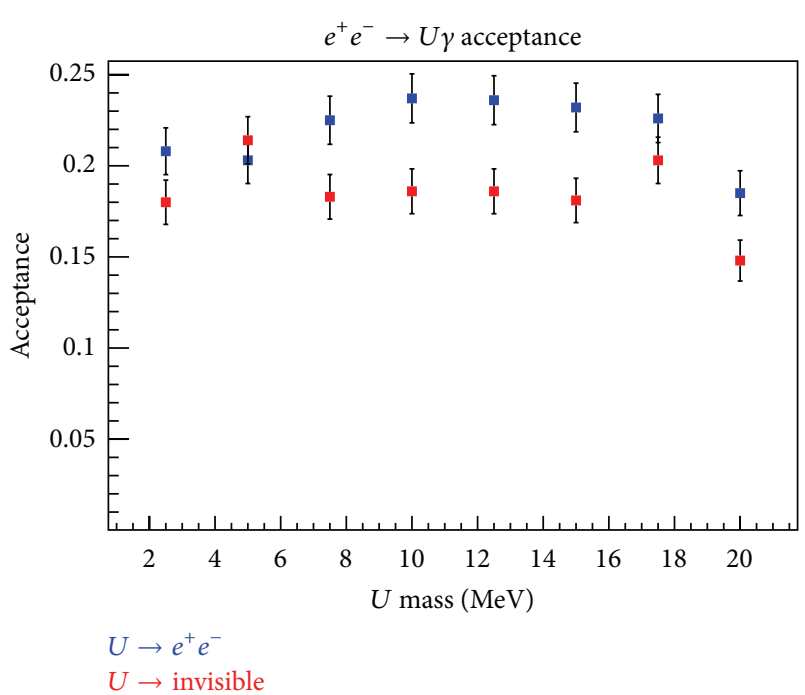

FIgURE 18: Acceptance for $U$ boson detection as a function of its mass.

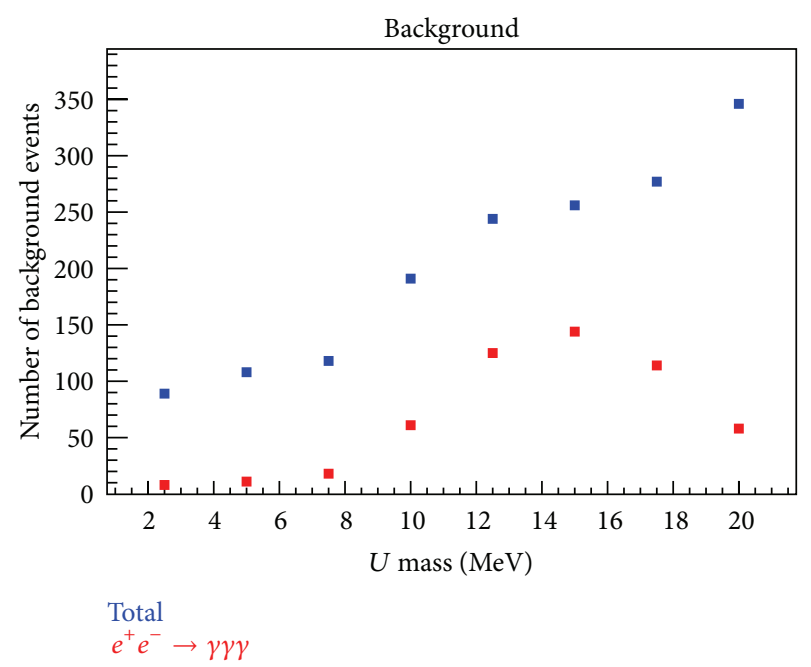

FIGURE 19: Background contribution as a function of the $U$ boson mass.

running. The expected sensitivity $\epsilon^{2} \sim 10^{-6}$ is common to both the visible and invisible $U$ boson decays and lies exactly in the region, preferred by the $g_{\mu}-2$ data, as seen in Figure 21. It partially overlaps with the result of the combination of the measurements of $\left(g_{e}-2\right)$ and $\alpha_{\mathrm{EM}}$, exploiting tenthorder calculations of the QED contributions. As discussed in Section 2, that region is still of high interest since no direct search for a $U$ boson has been performed accessing it and because of the recent shift by more than $7 \sigma$ [37] of the theoretical prediction for $g_{e}$ due to an error in the calculations. The proposed experiment could be the first to directly constrain the invisible channel. An upgrade of the BTF positron beam energy to $750 \mathrm{MeV}$ will extend the sensitivity to higher $U$ boson mass.

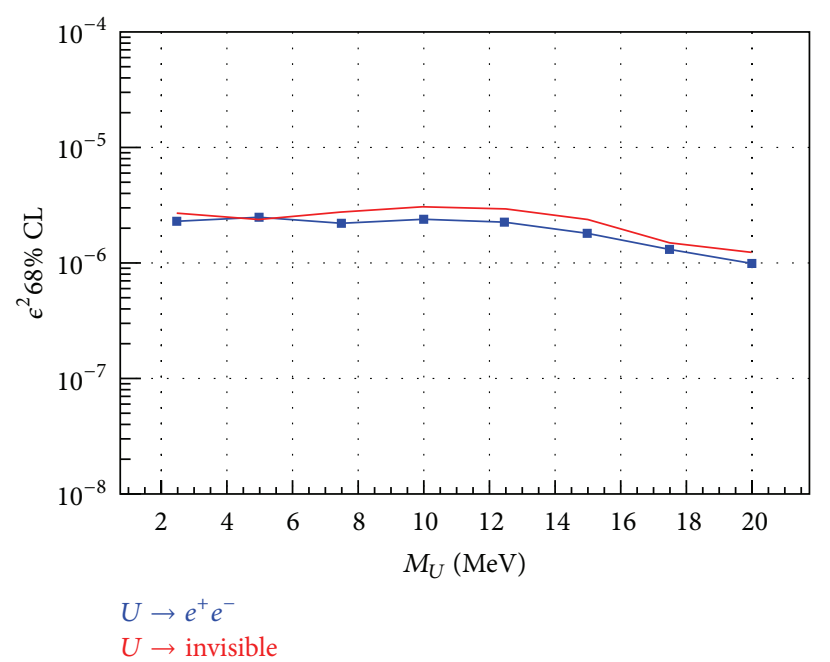

FIGURE 20: Expected exclusion limits in the $\epsilon-M_{U}$ plane in case of no signal.

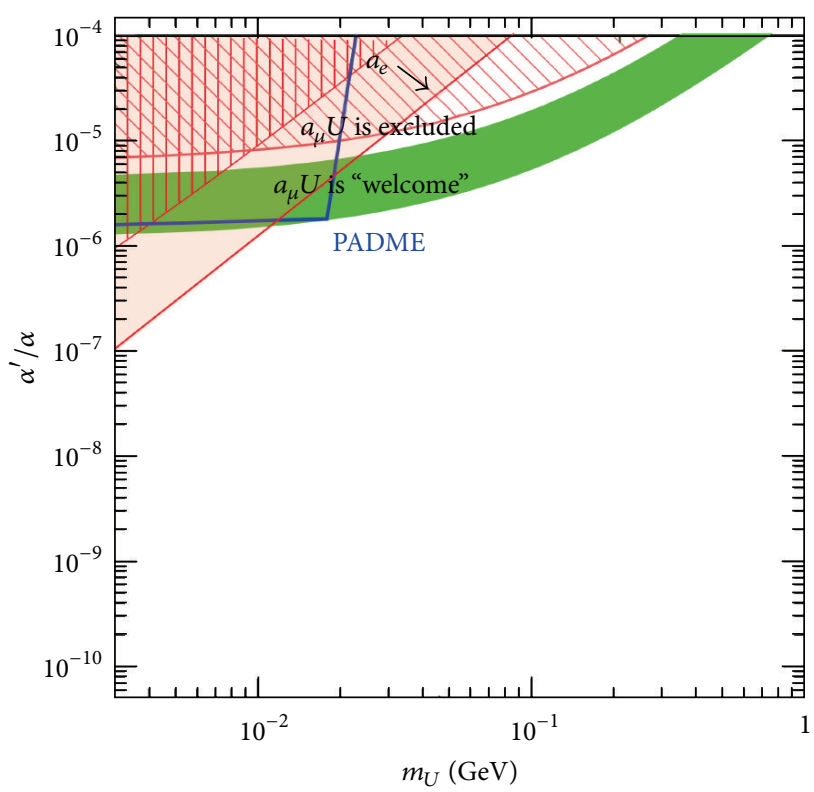

FIGURE 21: Expected exclusions in the invisible channel compared with the band of values preferred by current $g_{\mu}-2$ discrepancies.

At present there are a few experiments in different phases of preparations devoted to the search for dark photonsVEPP3 [38, 39], DarkLight [40], HPS [41], and BDX [42]. Among them, only VEPP3 is sensitive to invisible decays in the same region of parameter space. While the general approach is similar, there are few crucial differences. The VEPP3 proposal aims to use a storage ring which limits the beam energy to a fixed value of $500 \mathrm{MeV}$, while the DAФNE linac could be able to provide positrons with variable beam energy from $250 \mathrm{MeV}$ up to $750 \mathrm{MeV}$ and a beam spread of $1 \%$, thus extending the accessible region of $U$ boson masses to $\sim 27.5 \mathrm{MeV}$. Moreover, the presence 
of an active target allows the determination of the interaction vertex at the $\mathrm{mm}$ level, leading to a reduction of the background through improvement of the missing mass resolution. Another advantage of the experiment proposed in this paper is the relatively small calorimeter, since it is placed at a distance from the target about three times smaller with respect to the VEPP3 one, which minimizes the cost and timescale of the experiment. The presence of a magnetic spectrometer, apart from further reducing the background by measuring the momentum of the positrons that had emitted hard bremsstrahlung photons, could possibly allow the direct study of visible $U \rightarrow e^{+} e^{-}$decays. This channel makes the experiment sensitive to the bremsstrahlung production of $U$ bosons, thus pushing the accessible parameters region to higher masses. While providing an interesting benchmark possibility with the comparison of the results from visible and invisible searches done simultaneously, this falls outside the scope of the present paper and will be a subject of a future study.

\section{Conflict of Interests}

The authors declare that there is no conflict of interests regarding the publication of this paper.

\section{Acknowledgments}

The authors would like to thank Antonella Antonelli, Fabio Bossi, Paolo Valente, Matthew Moulson, Tommaso Spadaro, Sarah Andreas, and the LNF NA62 large angle veto group for encouraging those activities and for all the useful discussions. They would like also to thank the linac team at LNF for the information on present status and possible upgrade of BTF. The results were obtained exploiting the LNF computing facilities.

\section{References}

[1] B. Holdom and B. Phys. Lett, "Two U(1)'s and $\epsilon$ charge shifts," Physics Letters B, vol. 166, pp. 196-198, 1986.

[2] P. Galison and A. Manohar, “Two Z's or not two Z's?” Physics Letters B, vol. 136, no. 4, pp. 279-283, 1984.

[3] O. Adriani, G. C. Barbarino, G. A. Bazilevskaya et al., "An anomalous positron abundance in cosmic rays with energies 1.5100 GeV," Nature, vol. 458, pp. 607-609, 2009.

[4] M. Ackermann, M. Ajello, A. Allafort et al., "Measurement of separate cosmic-ray electron and positron spectra with the fermi large area telescope," Physical Review Letters, vol. 108, no. 1, Article ID 011103, 2012.

[5] M. Aguilar, G. Alberti, B. Alpat et al., "First result from the alpha magnetic spectrometer on the international space station: precision measurement of the positron fraction in primary cosmic rays of $0.5-350 \mathrm{GeV}$," Physical Review Letters, vol. 110, no. 14, Article ID 141102, 2013.

[6] O. Adriani, G. C. Barbarino, and G. A. Bazilevskaya (PAMELA Collaboration), "PAMELA results on the cosmic-ray antiproton flux from $60 \mathrm{MeV}$ to $180 \mathrm{GeV}$ in kinetic energy," Physical Review Letters, vol. 105, Article ID 121101, 2010.
[7] J. Beringer, J. F. Arguin, R. M. Barnett et al., "Review of particle physics," Physical Review D, vol. 86, no. 1, Article ID 010001, 2012.

[8] P. Fayet, "UA(1) symmetry in two-doublet models, $U$ bosons or light scalars, and $\psi$ and $\Upsilon$ decays," Physics Letters B, vol. 675, no. 2, pp. 267-271, 2009.

[9] A. Pukhov, Preprint INP MSU 98-41/542, http://arxiv.org/ pdf/hep-ph/9908288.

[10] A. Pukhov, "CalcHEP 2.3: MSSM, structure functions, event generation, batchs, and generation of matrix elements for other packages," http://arxiv.org/abs/hep-ph/0412191.

[11] S. Andreas, C. Niebuhr, and A. Ringwald, "New limits on hidden photons from past electron beam dumps," Physical Review D, vol. 86, Article ID 095019, 2012.

[12] E. M. Riordan, M. W. Krasny, K. Lang et al., "Search for shortlived axions in an electron-beam-dump experiment," Physical Review Letters, vol. 59, p. 755, 1987.

[13] J. D. Bjorken, S. Ecklund, W. R. Nelson et al., "Search for neutral metastable penetrating particles produced in the SLAC beam dump," Physical Review D, vol. 38, no. 11, pp. 3375-3386, 1988.

[14] A. Bross, M. Crisler, S. Pordes, J. Volk, S. Errede, and J. Wrbanek, "Search for short-lived particles produced in an electron beam dump," Physical Review Letters, vol. 67, no. 21, pp. 2942-2945, 1991.

[15] J. Schweppe, A. Gruppe, K. Bethge et al., "Observation of a peak structure in positron spectra from U+Cm collisions," Physical Review Letters, vol. 51, p. 2261, 1983.

[16] H. Merkel, P. Achenbach, and C. A. Gayoso (A1 Collaboration), "Search for light gauge bosons of the dark sector at the mainz microtron," Physical Review Letters, vol. 106, Article ID 251802, 2011.

[17] S. Abrahamyan, Z. Ahmed, APEX Collaboration et al., "Search for a new gauge boson in electron-nucleus fixed-target scattering by the APEX experiment," Physical Review Letters, vol. 107, Article ID 191804, 2011.

[18] F. Bossi, "Dark photon searches using displaced vertices at low energy $e^{+} e^{-}$colliders," Advances in High Energy Physics, vol. 2014, Article ID 891820, 8 pages, 2014.

[19] R. Essig, J. Mardon, M. Papucci et al., "Constraining light dark matter with low-energy $\mathrm{e}^{+} \mathrm{e}^{-}$colliders," Journal of High Energy Physics, vol. 2013, article 167, 2013.

[20] P. Adlarson, W. Augustyniak, W. Bardan et al., "Search for a dark photon in the $\pi^{0} \rightarrow e^{+} e^{-} \gamma$ decay," Physics Letters B, vol. 726, no. 1-3, pp. 187-193, 2013.

[21] F. Archilli, D. Babusci, D. Badoni et al., "Search for a vector gauge boson in $\varphi$ meson decays with the KLOE detector," Physics Letters B, vol. 706, no. 4-5, pp. 251-255, 2012.

[22] D. Babusci, D. Badoni, I. Balwierz-Pytko et al., "Limit on the production of a light vector gauge boson in $\phi$ meson decays with the KLOE detector," Physics Letters B, vol. 720, no. 1-3, pp. 111$115,2012$.

[23] B. Aubert, Y. Karyotakis, J. P. Lees et al., "Search for Dimuon decays of a light scalar boson in radiative transitions $\Upsilon \rightarrow \gamma A^{0}$," Physical Review Letters, vol. 103, Article ID 081803, 2009.

[24] M. Pospelov, "Secluded U(1) below the weak scale," Physical Review D, vol. 80, Article ID 095002, 2009.

[25] D. Hanneke, S. F. Hoogerheide, and G. Gabrielse, "Cavity control of a single-electron quantum cyclotron: Measuring the electron magnetic moment," Physical Review A, vol. 83, no. 5, Article ID 052122, 2011. 
[26] R. Bouchendira, P. Cladé, S. Guellati-Khélifa, F. Nez, and F. Biraben, "New determination of the fine structure constant and test of the quantum electrodynamics," Physical Review Letters, vol. 106, Article ID 080801, 2011.

[27] T. Aoyama, M. Hayakawa, T. Kinoshita, and M. Nio, "Tenthorder QED contribution to the electron $g-2$ and an improved value of the fine structure constant," Physical Review Letters, vol. 109, Article ID 111807, 2012.

[28] M. Diamond and P. Schuster, "Searching for light dark matter with the SLAC millicharge experiment," Physical Review Letters, vol. 111, Article ID 221803, 2013.

[29] R. Essig, J. A. Jaros, W. Wester et al., "Dark sectors and new, light, weakly-coupled particles," http://arxiv.org/abs/1311.0029.

[30] F. Hahn, F. Ambrosino, A. Ceccucci et al., "NA62: Technical Design Document," http://cds.cern.ch/record/1404985.

[31] E. Izaguirre, G. Krnjaic, P. Schuster, and N. Toro, "New electron beam-dump experiments to search for $\mathrm{MeV}$ to few-GeV dark matter," Physical Review D, vol. 88, Article ID 114015, 2013.

[32] G. Mazzitelli, A. Ghigo, F. Sannibale, P. Valente, and G. Vignola, "Commissioning of the DAФNE beam test facility," Nuclear Instruments and Methods in Physics Research A: Accelerators, Spectrometers, Detectors and Associated Equipment, vol. 515, no. 3, pp. 524-542, 2003.

[33] P. Valente, B. Buonomo, and L. Foggetta, "Possible upgrades of the DAFNE Beam-Test Facility (BTF)," INFN-14-06/LNF, 2014.

[34] B. Buonomo et al., DAFNE note in preparation, 2014.

[35] W. Heitler, The Quantum Theory of Radiation, Clarendon Press, Oxford, UK, 1954.

[36] G. Eigen, Z. Zhou, D. Chao et al., "A LYSO calorimeter for the SuperB factory," Nuclear Instruments and Methods in Physics Research Section A: Accelerators, Spectrometers, Detectors and Associated Equipment, vol. 718, pp. 107-109, 2013.

[37] T. Aoyama, M. Hayakawa, T. Kinoshita, and M. Nio, "Revised value of the eighth-order contribution to the electron $g-2$," Physical Review Letters, vol. 99, Article ID 110406, 2007.

[38] B. Wojtsekhowski, "Searching for a U-boson with a positron beam," AIP Conference Proceedings, vol. 1160, pp. 149-154, 2009.

[39] B. Wojtsekhowski, D. Nikolenko, and I. Rachek, "Searching for a new force at VEPP-3," Preprint JLAB-PHY-12-1597, http://arxiv.org/abs/1207.5089.

[40] M. Freytsis, G. Ovanesyan, and J. Thaler, "Dark force detection in low energy ep collisions," Journal of High Energy Physics, vol. 2010, article 111, 2010.

[41] The Heavy Photon Search Collaboration (HPS), https://confluence.slac.stanford.edu/display/hpsg/.

[42] M. Battaglieri, A. Celentano, R. de Vita et al., "Dark matter search in a Beam-Dump eXperiment (BDX) at Jefferson lab," http://arxiv.org/abs/1406.3028. 

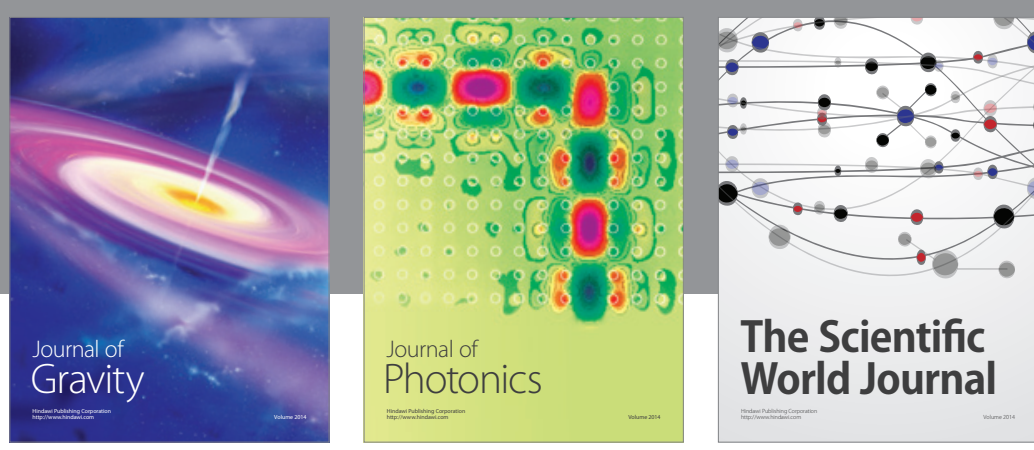

The Scientific World Journal
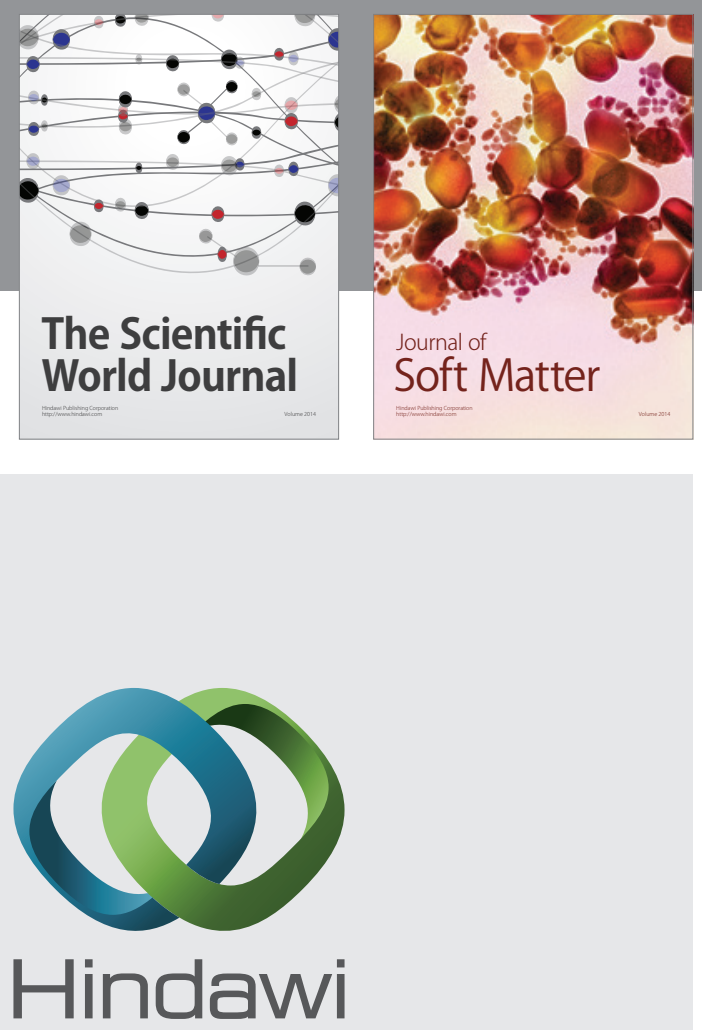

Submit your manuscripts at

http://www.hindawi.com

nternational Journal of

Statistical Mechanics
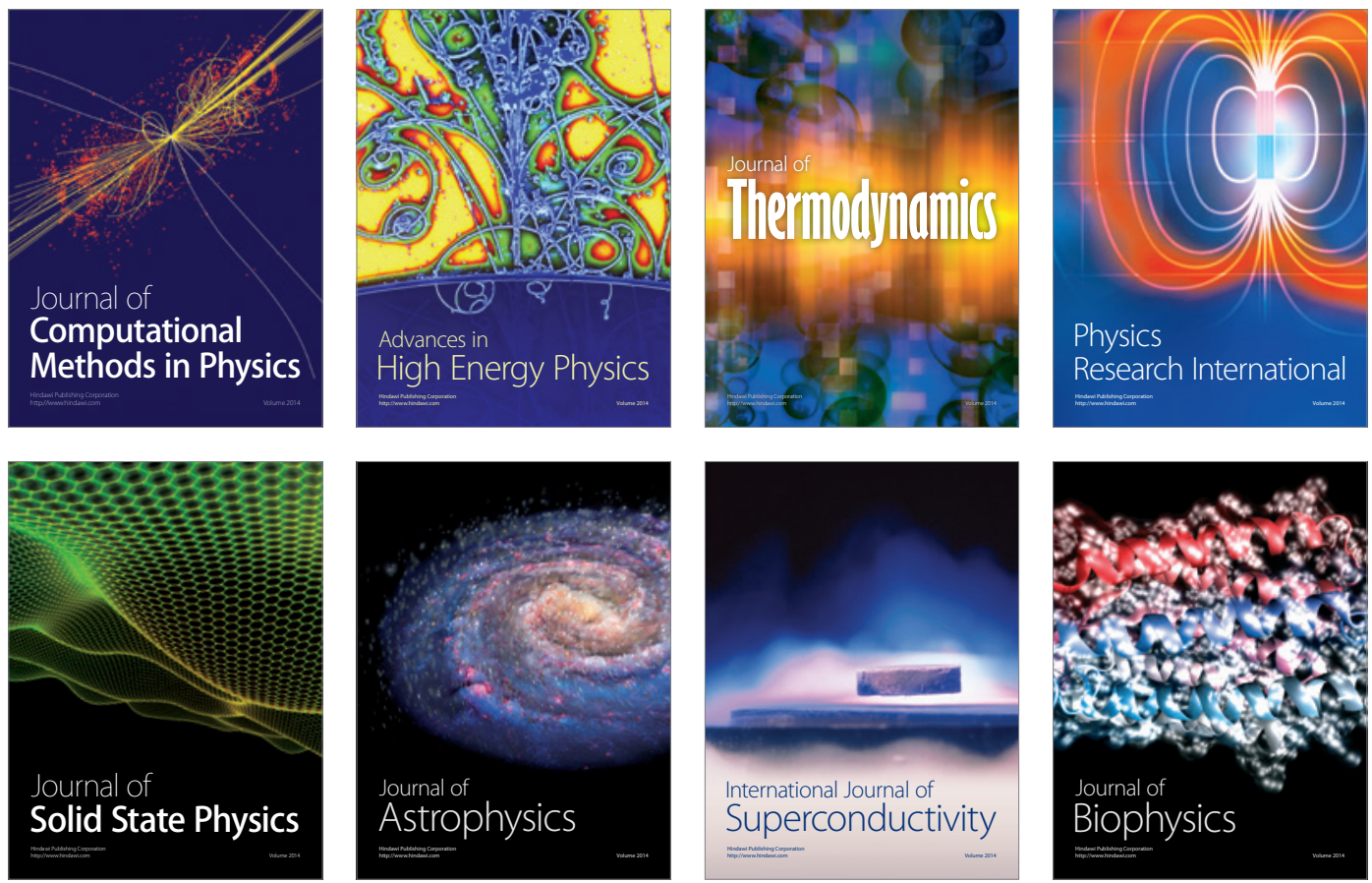
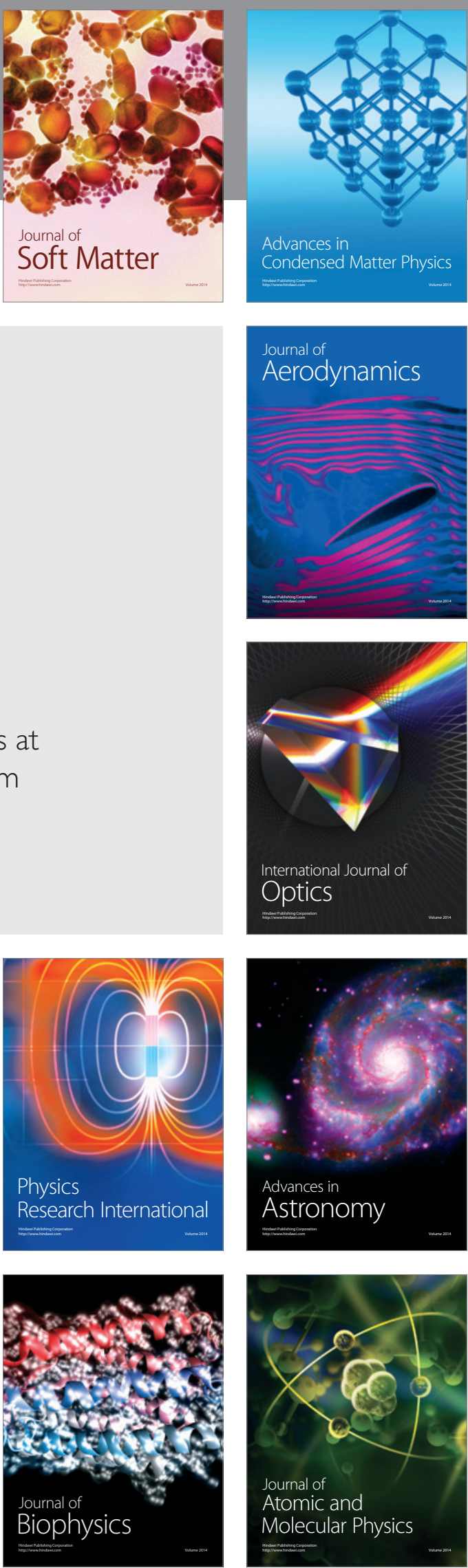